\title{
Mobile Broadband Public Safety Grade Network and Counter Remotely Piloted Aircraft Systems
}

\author{
Marian Buric \\ Protection and Guard Service \\ Bucharest, Romania \\ marianb61@gmail.com
}

\begin{abstract}
An effective Counter Remotely Aircraft System is a major objective of many researchers and industries entities. Their activity is strongly impelled by the operational requirements of the Law Enforcement Authorities and naturally follows both the course of the latest terrorist events and technological developments. One of the key issue regarding the operational requirements is deploying of a Counter Remotely Aircraft System over metropolitan area which seems to be one of the most attractive target for terrorists. Technical architecture of a Counter Remotely Aircraft System is a complex one, consists from numerous sensors, distributed processors, neutralization elements and command and control room, spread over a large area. All these elements need to be interconnected using an appropriate state of the art error free network, capable to manage in real time and secure all data. This paper starts up from a complete model for a Counter Remotely Aircraft System and proposes a Mobile Broadband Public Safety Grade Network as solution for information flow between its architectural components.
\end{abstract}

Keywords-Counter Remotely Piloted Aircraft Systems; Mobile Broadband Public Safety Grade Network; Public Safety; RPAS.

\section{INTRODUCTION}

The main goal of this paper is to propose a synergy between two key issues intimately connected to Law Enforcement Authorities' missions: Counter Remotely Piloted Aircraft System $^{1}$ (C-RPAS) and a Mobile Broadband Communications specific for Public Safety. Both of them provide functions and services needed for prevention and protection from events that could endanger the safety of general public and the society.

There is a continuous increasing of number of the RPASs applications in commercial and non-commercial fields (e.g., industry, agriculture, services, research, scientific, governmental non-military and so on) but unfortunately in the same time the RPASs have been becoming a really threat and weapon in nowadays asymmetric warfare, terrorist attacks or malicious uses [1].

Mitigating the threats of illegal use of the RPASs envisages both legal and technological aspects. On the legislation side, the aim is RPASs integration into non-segregated airspace in

This work was supported in part by the European Union's Horizon 2020 research and innovation programme under grant agreement $N^{\circ} 700643$.

${ }^{1}$ A Remotely Piloted Aircraft System is composed from Remotely Piloted Aircraft (RPA) and associated Remotely Control Unit (RCU).

978-1-5386-2350-3/18/\$31.00(2018 IEE multi-aircraft environment (including manned vehicles). The technological aspect aims to adapt and provide the technologies necessary to avoid mid-air collisions and to make RPAS technology compliant with national and international agreed aviation certification standards. Nevertheless, as a last radical solution it is imperious to design an effective counter RPASs according to an acknowledged standard.

The RPASs are used for civil or military goals. This paper takes into account only RPASs which were designed to be used by civilians in recreational or commercial applications. There are many criteria for RPASs classification according to principle of flying and configuration, flight characteristic and handling, autonomy, endurance, kinetic energy, maximum weight or payload capacity, purpose and so on. This paper takes into account low, slow, small (LSS) RPASs, fixed wing and rotary-blade RPASs, up to $20 \mathrm{~kg}$ MTOM, glider, quadcopter or jet turbine types, according to European ${ }^{2}$ and American classification [2].

In the second chapter, there is a briefly description of an effective complete C-RPAS architectural model following step by step a five "kill chain" from detection up to forensic stage. More information about the rationale of this model could be found in [3]. Besides the description of this model there are highlighted information flow between its elements, information type and requirements for throughput, latency, error rate, priority and so on.

In the third chapter, taking into account a metropolitan area with its specifically operational and technical requirements, it is proposed a practical solution for deploying the C-RPAS model described in previous chapter using as data carrier between its architectural components a mobile broadband network with embedded feature for Public Safety applications. There are highlighted the arguments for chosen solution as response to all requirements which have imposed on previous chapter. In the same time, there are emphasized the benefits of using a dedicated mobile broadband for public safety and which of its features are appropriate for Law Enforcement Authorities (LEA) specifically applications.

\section{COUNTER REMOTELY PILOTED AIRCRAFT SYSTEM}

LEAs need a C-RPAS with not only theoretically but also

${ }^{2}$ www.ultraconsortium.eu 
practically nearly $100 \%$ demonstrated successful rate.

C-RPAS systems, in order to fulfil all their operational functionalities, must work according to a "kill chain" model. A Sandia National Labs report defined a three step "kill chain" model: detection, classification and neutralization. For a complete C-RPAS model it is necessary, according to author opinion [3], to add other two phases: tracking and forensic. Therefore, the model has been becoming a five "kill chain" model: detection, classification, tracking, neutralization and forensic. An effective C-RPAS system must successfully carry out each step strictly in this chain order (Fig. 1) [3].

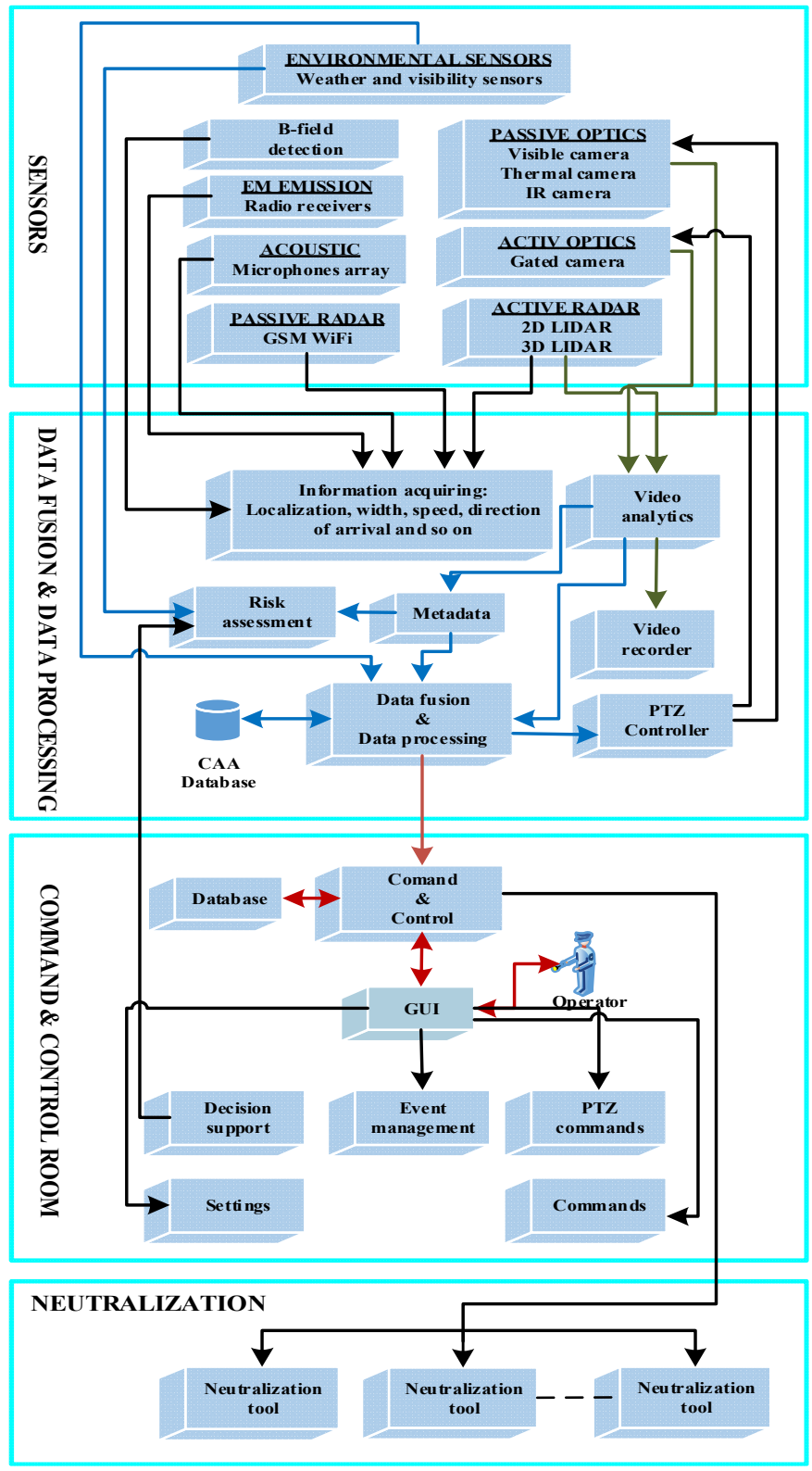

Fig. 1. C-RPAS architectural concept according to [3]

\section{A. Detection}

In this phase, a large number of heterogeneous sensors organized into a sensors network are collecting information from a cluttered noisy background environment data. The sensors must be deployed in accordance with their effective range, with the aim to defeat the whole protected zone (named also the responsibility zone of the C-RPAS). The responsibility zone is defined in $3 \mathrm{D}$ dimensions with a halfsphere shape.

In order to be effective, the sensors used in detection phase must complement with neutralization and tracking phases.

It is necessary to use a complete mix of sensors because:

- There are a large variety of RPASs which must be detected, with very different characteristics (e.g., cross section, speed, acoustic and radio signature, flying mode). Consequently, there is not a single sensor type appropriate to any possible malicious flying object. Sandia National Laboratories has done an evaluation of effectiveness of different sensor types according to three types of RPAS - glider, quadcopter and jet turbine [2]. Reference [3] has completed the list with a new state-of-the-art one which is a passive radar that is using "electro smog".

- There is a cluttered video, audio and spectral radiofrequencies background and consequently a mix between a lot of sensors boost the successful rate and lower the false positive and false negative rates [4].

- The attackers could use some measures with intent to impede the successfully detection of the malicious RPAS like using of the cellular communication or nonstandard frequencies for Command and Control (C2) link, fully autonomous mode and so on.

- In order to be full effective and to mitigate the threat at a high level, the C-RPAS solution must be addressed to both the RPAs and their Remote Controller Units (RCUs).

The most challenging issue for detection phase is that the amount of data required to provide a reasonable response time is very large. Each sensor should assign a unique ID event and timestamp and then provide valuable information for classification and risk assessment (e.g., azimuth, elevation, distance, speed, propulsion, trajectory, video and so on).

\section{B. Classification}

Data that has been collected by all sensors in detection phase is combined and processed by a specialized algorithm (into "Data fusion \& Data processing" unit from Fig. 1) following strictly one by one three steps in order to decide if de object detected is or not a RPAS, if the object identified as a RPAS represent or not a threat and finally the target's level priority.

The environmental background scanned by sensors in detection phase is a cluttered and noisy one where there are a mix of multiple objects which could be easily confused with a RPAS. Therefore, the first task of the C-RPAS, in classification phase, must be to decide if an object into the responsibility zone of the C-RPAS is or not a RPA. Failure to correctly classify an object in this step may result in a false 
positive or false negative classification with an according implying in the following steps of this phase and in the following phases - tracking and neutralization.

Not all RPAs crossing a perimeter should be automatically treated as hostile or malicious. Moreover, a C-RPAS system may be installed not only in "no fly zones". Once an object has been identified as RPA, it must be evaluated and classified further as a threat or not. This process could be done through a preliminary assessment and decision that RPAS is or not an authorized one. This decision is taken after interrogation the Civil Aviation Authority (CAA) database. The object validation as a threat must be done firstly by machine, confirmed by a human operator, labeled accordingly and displayed into a GUI. Moreover, as feasible solution for RPASs validation could be used Proximity Service (ProSe) described in the following chapter. In this way a communication is established between registered RPASs and C-RPAS's sensors and finally RPASs will be accordingly classified.

\section{RPAS risk level assessment}

If the object was classified as a threat, it need further to be assessed for a risk level in order to take the most appropriate response at a multi option neutralization phase. Sometimes the safety risk level of the effects of the counter measures could be higher than the potential risks of the offending RPAS. Moreover, there are scenarios when in responsibility area of a C-RPAS could be detected and confirmed as threats multiple heterogeneous RPASs with different parameters. Therefore, it is necessary to carry out a risk assessment process and finally to determine the risk level of each target. The level of the risk should be automatically calculated based on certain parameters and then write it down on target's label on the GUI. Risk level could be calculated according to the type of target (e.g. glider, quadcopter, jet), direction of arrival, range (distance from the target to the point of interest or to the protected area), velocity (known also as speed), estimated time of arrival, number of the targets that have been detected and are tracking in the same time, number of the sensors that have confirmed the target, altitude, interrogation results of other data bases (e.g. CAA) and target's size.

\section{Tracking}

At the fusion level, "handover" procedures will be implemented to properly track the targets crossing more than one sensor set (the alarm will be then displayed on the GIS map with its tracking path and target ID). Information about the target type and its level of threat will be also given, by applying methodologies from previously classification phase.

In order to get more time for the following phases, an initial coarse tracking will be put in place. A continuous process based on anomaly detection theory, statistical signal processing coupled with well-defined rules for intruder behavior analysis will be used. The goal is to discriminate, among the detected events, between normal or anomalous behaviors (forbidden trajectories, violation of flight regulations, suspect patterns). Finally, once a threshold for information necessary have been acquired and processed, a fine tracking will be implemented.

Handover procedures must take place automatically, and information about the active system(s) will be reported on the GUI. The full track is recorded for post-incident analysis.

\section{E. Neutralization}

The goals of the neutralization phase are denying RPAS's mission (mostly non-destructive) or RPAS's neutralization (mostly destructive). Based on the results of classification phase, and specially on results of risk assessment, an intelligent C-RPAS should dynamically chose to carry out the appropriate method to meet one of the aforementioned goals.

The neutralization method need also to be chosen according to the environment and to the effect of the method (e.g., destructive or non-destructive). There are a lot of neutralization solutions each of them with its own weakness and strength points depending on technical implementation, goals and its applicability [3]: geofencing, physical or kinetic solution, jamming and hacking. Moreover, there is a correspondence between RPAS's capabilities and the appropriate neutralization solutions. Reference [3] has scaled the level of threaten for each RPAS's capability apart and the estimated level of effectiveness for each neutralization solution correspondingly. There are few interesting observations on [3]:

- For each RPAS's capability there are few neutralization solutions with different level of effectiveness.

- The effectiveness depends largely on how accurate detection phase was and the time at disposal for neutralization.

- The evidences from forensic phase greatly depend on results of the neutralization phase (i.e., its destructive effects).

- It seems that is necessary more than one neutralization solution in the same time like in detection phase when all types of sensors are needed.

\section{F. Forensic}

The forensic is logically the last phase of a C-RPAS process which have to meet the following objectives: establish who is the owner of the RPAS and for what purpose it was used for, retrieve the flight path and the home point and retrieve valuable information from application databases installed into remote controller and mobile device.

In the first step, it is recommended to obtain a forensic image of all system and memories files from all RPAS components. In order to conclude what file has been modified it is obviously that we must know the initial state of these files.

The forensic activity envisages the following RPAS components [5], [6]: RPA, sensors, remote controller and 
mobile device.

First of all, it is necessary to evaluate physically all components of the RPAS and note down the type of each component, serial number, payload, sensors, or any evidence which could point out for what purpose have the RPAS been used on.

The main elements of the RPA which need to be investigated are GPS and Flight Controller. Through GPS are received geographic coordinates from satellites which then are transmitted to the Flight Controller and camera.

The most important information and artefacts which could be retrieved from Flight Controller are related to OS, Flight Controller ID, flight data records (e.g., home position, flight path), data about RPA components (e.g., motor load, motor speed, battery load, battery voltage, system failure), information about boot sequence, maintenance and logistic.

The types of sensors which have been found attached to RPA tell us about the purposes of the flight (e.g., optical, thermal, LIDAR, NIR, WiFi). From sensors could be exported images files, video files and EXIF data. EXIF data point out information about the camera and where the pictures were taken through geographic coordinates and altitude therefore rebuilding a 3D scene. The EXIF data are persistent even after the RPA has crashed.

From Remote Controller application memory, we could retrieve some artefacts like vendor applications, owner name and account, default settings, launch points, dates, association of geographical coordinates to GPS data from media, mission plan and flight telemetry data, connection logs to cloud services and user credentials for log to cloud, and so on.

Usually between the Remote Controller and human operator there is a mobile device or tablet. Into mobile device there are also a lot of valuable information, like OS, application for settings and control the flight, subset of data recorded on the Flight Controller, imagines, video and Flight Controller ID.

A counter forensic should not be neglected in order to determine if the integrity of evidence has been modified or the time stamp was tampered.

There are web sites were files retrieved from RPAS's components could be sent for online parser ${ }^{3}$.

\section{MOBILE BROADBAND NETWORK AS SUPPORT FOR A COUNTER REMOTELY PILOTED AIRCRAFT SYSTEM}

\section{A. C-RPAS requirements regarding a Mobile Broadband Public Safety Grade Network}

A C-RPAS model, as highlighted on chapter two, must be effective in any operational environments where it is expected to be deployed (i.e. metropolitan areas, critical infrastructures, border areas). Particularly for metropolitan area where a $\mathrm{C}$ RPAS deployment and its architectural components interconnection could be tremendous tasks, this paper proposes as support a Mobile Broadband Public Safety Grade

\footnotetext{
${ }^{3}$ https://airdata.com/
}

Network based on LTE technology. There is a standardization roadmap for this solution and now there are running the last steps. Moreover, there have been already designed and are deploying the first Mobile Broadband Communications for Public Safety (e.g. FirstNet ${ }^{4}$ in USA).

The correspondence between C-RPAS requirements and the Mobile Broadband Public Safety Grade Network capabilities are succinctly pointed out on following table.

\section{TABLE I. TECHNICAL REQUIREMENTS}

\begin{tabular}{|c|c|}
\hline $\begin{array}{l}\text { C-RPAS's } \\
\text { requirements }\end{array}$ & Network features \\
\hline $\begin{array}{l}\text { Data and video } \\
\text { information flow } \\
\text { between sensors and } \\
\text { Data fusion and Data } \\
\text { processing unit at a } \\
\text { maximum } 1 \mathrm{Mb} / \mathrm{s} \text { for } \\
\text { each pair of } \mathrm{UEs}^{5} \text {. }\end{array}$ & $\begin{array}{l}\text { Throughput depends on Sidelink configurations } \\
\text { (i.e. number of Resource Blocks, Sidelink period, } \\
\text { PSCCH duration, Modulation and Coding } \\
\text { Scheme). Hence, the throughput may vary } \\
\text { between } 1 \mathrm{Mb} / \mathrm{s} \text { and } 3 \mathrm{Mb} / \mathrm{s} \text { for } 10 \text { UEs, no more } \\
500 \mathrm{~m} \text { between UEs [8]. }\end{array}$ \\
\hline $\begin{array}{l}\text { Preponderant traffic } \\
\text { from sensors towards } \\
\text { Data fusion and Data } \\
\text { processing unit. }\end{array}$ & Symmetric UL/DL usage pattern [7]. \\
\hline $\begin{array}{lrr}\text { A high level } & \text { of } \\
\text { availability } & & \text { and } \\
\text { resilience. } & & \\
\end{array}$ & $\begin{array}{l}\text { Network availability at least } 99.99 \% \text { of the time } \\
\text { (i.e. less than } 50 \mathrm{~min} / \text { year of unplanned outages). }\end{array}$ \\
\hline $\begin{array}{l}\text { Multimedia group } \\
\text { communications. }\end{array}$ & $\begin{array}{l}\text { Group communications system enablers } \\
\text { (MCPTT) with the following characteristics [9]: } \\
\text { O Besides Push To Talk (PTT) application, } \\
\text { multimedia group communications (voice, } \\
\text { video and data). } \\
\text { O End-to-end set-up time less than or equal to } \\
300 \text { ms (for an uncontended network where } \\
\text { there are no presence checking and no } \\
\text { acknowledgements requested from receiver } \\
\text { group member). } \\
\text { The end-to-end delay for media transport for } \\
\text { group communications should be less than or } \\
\text { equal to 150 ms. } \\
\text { Priority and pre-emption features. } \\
\text { Scalable multiple groups configuration. } \\
\text { Interaction with proximity-based services. } \\
\text { O High availability of group communication. } \\
\text { The same content sent to a large number of } \\
\text { subscribers at the same time through } \\
\text { Multimedia Broadcast Multicast Service } \\
\text { (MBMS) }\end{array}$ \\
\hline $\begin{array}{l}\text { Proximity-based } \\
\text { services (devices are } \\
\text { sensing each other). }\end{array}$ & $\begin{array}{l}\text { ProSe Discovery (Evolved Packet Core (EPC) } \\
\text { level discovery, operates on uplink spectrum with } \\
\text { impact on the LTE capacity less than 1\%) and } \\
\text { ProSe Communication (eNB locally routed } \\
\text { communication), a full 'operator-centric' } \\
\text { approach that permit to exploit all ProSe } \\
\text { capabilities. } \\
\text { Support for ProSe Group Communication, ProSe } \\
\text { Broadcast Communication, ProSe UE-to-Network } \\
\text { Relay, ProSe UE-to-UE Relay. }\end{array}$ \\
\hline \begin{tabular}{lr} 
A high reliability \\
network & including \\
isolated & network \\
operation (fall backhaul \\
\multicolumn{2}{c}{ connections). }
\end{tabular} & $\begin{array}{l}\text { eNB acts as a radio resources manager, locally } \\
\text { routed communications for Public Safety UEs. } \\
\text { eNB as application server. } \\
\text { ProSe capabilities as response to backhaul fall. }\end{array}$ \\
\hline C-RPAS is a mission- & Access priority at Access Stratum (AS) level, \\
\hline
\end{tabular}

${ }^{4}$ www.firstnet.gov

${ }_{5}^{5}$ User Equipment means a conventional terminal like a smartphone but also any sensor with embedded USIM

${ }^{6} \mathrm{PSCCH}-$ Physical Sidelink Control Channel 
critical system and through control of UE cell selection and accordingly the reselection procedures, Access Class Barring information flow from (ACB), to prevent overload of the access channel, sensors towards Data Enhanced Access Control by 'barring rates', fusion and Data Service Specific Access Control and Extended processing and further Access Barring, a mechanism to restrict network toward Command and access for low priority devices.

Control Room and then Admission priority at Non Access Stratum (NAS) towards neutralization level, based on Allocation and Retention Priority units must be prioritized (ARP) parameter (15 priority levels with preand traffic guaranteed.

emption and pre-emptability flags added), through default admission priority settings and dynamic admission priority settings.

Data plane QoS (latency, error rate and throughput), through QoS Class Identifier (QCI) and Guaranteed Bit Rate/Maximum Bit Rate (GBR/MBR) parameters for GBR bearers.

Per-Packet Priority for ProSe service [10].

Build upon the security foundations of the previous cellular systems.

Confidentiality, integrity protection and replay protection to NAS and Radio Resource Control (RRC) signaling.

User plane confidentiality protection at Packet Data Convergence Protocol (PDCP) layer [11]

Robust, certified and AES, SNOW 3G and ZUC algorithms and proved security system-wide upgrade from 128-bits to 256-bits solutions authentication, for

confidentiality and data integrity.

keys.

Ciphering indicator feature.

Security based on IETF defined IPSec security protocols as specified in RFC-4301 and in RFC2401, for backhaul and EPC domain.

Tamper resistant removable storage UICC/USIM. Temporary identities for individual and group users (long term information like IC, IMEI and IMSI are not stored within a security context).

Over-the-air security profile control and configuration.

High-power terminals, besides Class 3 with a Extended range of maximum transmit power of $23 \mathrm{dBm}$, it was operation. $\quad$ defined Class 1 for Band 14 with a maximum transmit power of $31 \mathrm{dBm}$ [7].

Dynamically changing Over-the-air change of the UE's configurations of UEs configurations and profiles (embedded in USIM) like and parameters. prioritization, QoS, security, access and subscription profiles, group allocations.

Mobile Broadband Communications for Public

Safety can be utilized by almost any application relying on IP communications.

The mobile network There are delivery options for Mobile Broadband should be a transparent Communications (e.g. using dedicated networks, carrier service. designed as 'Public Safety Grade" commercial networks and hybrid solutions).

$\mathrm{RAN}^{8}$ sharing enhancements (including national roaming).

Taking into account all features summarized in the above table, there are enough reasons to consider a Mobile Broadband Public Safety Grade Network as support for a CRPAS deployment in a metropolitan area.

\section{B. Mobile Broadband Public Safety Grade Network as carrier provider for $C$ - RPAS}

In Fig. 2 there is an example of C-RPAS deploying using as interconnection between its elements ProSe capabilities of a Mobile Broadband Public Safety Grade Network based on

\footnotetext{
${ }^{7}$ NPTSC, 'Defining public Safety Grade System and Facilities', May 2014

${ }^{8}$ RAN - Radio Access Network
}

LTE technology developed according to 3GPP standards. There are shown all network components involved in information flow on both user and control planes in a Home Public Land Mobile Network (HPLMN). The sensors and Data Fusion and Data Processing unit are deployed in Universal Terrestrial Radio Access Network (U-TRAN) domain while the C-RPAS application server in external Packet Data Network (PDN) domain on LEA's premises.

UEs representing each sensor, construct a Fully Qualified Domain Name (FQDN) using the HPLMN ID and relies on DNS translation to obtain the IP address of ProSe Function and then register with ProSe Function sending a UE Registration Request message over PC3 interface. If there is no associated UE context, the ProSe Function gets the subscription information for restricted ProSe Direct Discovery and ProSe Direct Communication from Home Subscriber Server (HSS) over PC4a interface. As response to UE Registration Request messages, ProSe Function generates EPC ProSe User IDs for the authorized UEs (EPUID_1, EPUID_2 ... EPUID_n), stores them together with users' IMSI and responds to UEs by sending UE Registration Response messages.

The second step consists in C-RPAS application registration for ProSe service. Prior to ProSe registration, each sensor's UE has received an Application Layer User ID (e.g. ALUID_1 for UE1). ALUID represents an identity identifying a user within the context of a specific application, here for C-RPAS application. Each UE sends Application Registration Request (e.g. EPUID_1, Application ID, ALUID_1 for UE1) message to ProSe Function. Application ID is used to identify C-RPAS application on external PDN. ProSe Function uses EPUIDs of the sensors UEs to retrieve users' profiles, checks that the requested application is on the stored list of authorized Application IDs and sends a ProSe Registration Request (ALUIDs, EPUIDs, PFID) message to the ProSe Application Server indicating that users of this application (identified as ALUIDs) have requested to use ProSe for that application. PFID is the ProSe Function ID. If the ProSe Application Server accepts the request, it stores the users' Application Layer User ID (ALUIDs) and EPC ProSe User ID (EPUIDs) together with the PFID and then sends a ProSe Registration Response message to ProSe Function. Following ProSe Registration Response message from ProSe Application Server, ProSe Function send Application Registration Response messages to UEs indicating that the registrations were completed.

In order to be alerted and control the proximity with sensors' UEs, Data Fusion and Data Processing UE (UE_D), triggers the Proximity Request procedure. UE_D sends a Proximity Request (EPUID_D, Application ID, ALUID_D, ALUIDs sensors' UEs, window, Range, D's location) message to ProSe Function. The window parameter indicates the time period during which the request is valid. Range is a requested range class for this application chosen from the set of allowed range classes. D's location is the current location of UE_D 
with the best accuracy known by UE A. Further, ProSe Function sends a Map Request (ALUID A, ALUID 1), (ALUID_A, ALUID_2),.. (ALUID_A, ALUID_n) to the ProSe Application Server where UE_D' application-specific ProSe permissions are checked. ProSe Function, based on message received from ProSe Application Server acknowledges the proximity request to UE_D.

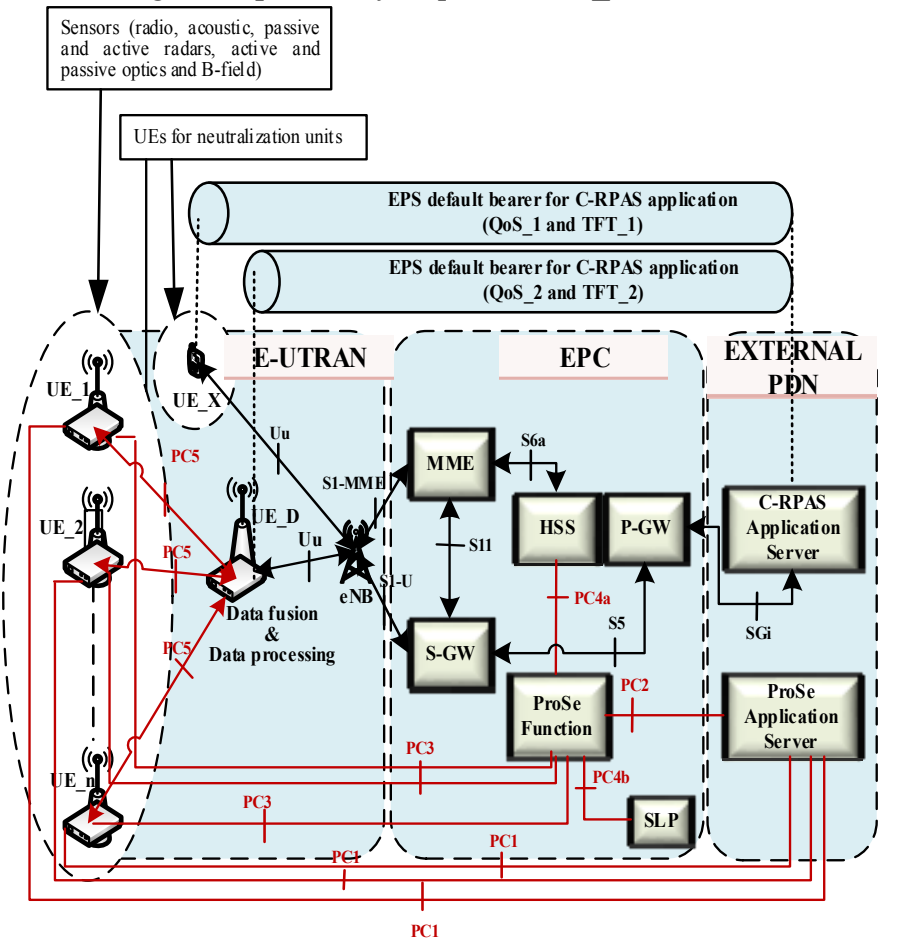

Fig. 2. C-RPAS architectural components deployed into Mobile Broadband Public Safety Grade Network

The sensors (UE1...UEn), are pre-configured with authorization information and authorized to perform restricted ProSe Discovery and ProSe Communications and addition information regarding out-coverage operation from ProSe Function over PC3 interface. To note that C-RPAS application being a Public Safety application, it requires restricted ProSe Discovery only that takes place with explicit permission from the sensors and data fusion and processing that are being discovered. Furthermore, it will be used restricted ProSe Discovery Model B. In order to engage a restricted ProSe Discovery, the ProSe Function of the HPLMN assigns a ProSe Discovery UE ID and sends it to the UE. If there is no associated UE context, the ProSe Function gets the subscription information for restricted ProSe Direct Discovery and ProSe Direct Communication from HSS over PC4a interface.

To perform restricted ProSe Discovery announcing and monitoring over PC5 interface, sensors get from ProSe Function ProSe Query Codes and ProSe Application Masks which in turn are requested and received from Application Server over PC2 interface.

One default EPS bearer service is established between Data
Fusion and Data Processing unit and C-RPAS Application Server with some specific parameters related to QoS (i.e. QoS Class Identifier, Allocation and Retention Priority, Guaranteed Bit Rate and Maximum Bit Rate) and Traffic Flow Template which enroll IP traffic filters (i.e. source and destination IP address, source and destination port as well as protocol identifier). In the same way, another default EPS bearer with specific parameters is established between C-RPAS Application Server and UE_X which represents neutralization tools.

\section{CONCLUSIONS AND FUTURE WORKS}

It is obviously that a Mobile Broadband Public Safety Grade Network designed accordingly to 3GPP standards, could be used as a transparent network infrastructure for a large Public Safety applications number. Even in the field of C-RPAS solutions could be used applications to check the RPASs complying with the rules on aeronautical safety, air traffic management, pilot licensing and aeronautical certification of the aircrafts.

A potential future work consists in studying the possibility of using ProSe service for interrogating CAA database about status of RPASs within non-segregate space.

There is also an emergent need to create a strong C-RPAS community which gather people of the main fields: LEA, research and industry. This paper has taken into account the need to strengthen the existing C-RPAS community and to offer them a potential solution for information flow between architectural components of a C-RPAS.

\section{REFERENCES}

[1] David Kovar, "Defending Against UAVs Operated by Non-State Actors", The Fletcher School, Tufts University, Final Thesis, GMAP 16, https://integriography.wordpress.com/2017/03/24/defending-againstuavs-operated-by-non-state-actors/

[2] Gabriel Birch, John Griffin, and Matthew Erdman, "UAS Detection, Classification, and Neutralization: Market Survey 2015," Sandia National Laboratories, 2015, http://prod.sandia.gov/techlib/accesscontrol.cgi/2015/156365.pdf.

[3] Marian Buric and Geert De Cubber, "Counter remotely piloted aircraft systems", in MTA review, vol. XXVII, No. 1, Jun. 2017, pp. 9-18.

[4] The NATO Industrial Advisory Group Study SG-170, "The Engagement of Low, Slow and Small Aerial targets by GBAD," July, 2013.

[5] M. Maarse and L. Sangers, "Digital forensics on a DJI Phantom 2 Vision+ UAV," University of Amsterdam, Apr. 29, 2016.

[6] D. Kovar, G. Dominguez, and C. Murphy, "UAV (aka drone) Forensics," SANS DFIR Summit Jun. 23-24, 2016.

[7] Ramon Ferrus, Oriol Sallent, "Mobile broadband communications for public safety: the road ahead through LTE technology", Willey, 2015.

[8] Richard A. Rouil, Fernando J. Cintron, Aziza Ben Mosbah, Samantha M. Gamboa Quintiliani, "Implementation and Validation of an LTE D2D Model for ns-3", NIST publications, 2017

[9] 3GPP TS 23.303: "Technical specification group services and system aspects; Proximity-based services (ProSe)", Release 15, 2017.

[10] 3GPP TS 33.303: "Technical specification group services and system aspects; Proximity-based services (ProSe); Security aspects”, Release 14, 2017.

[11] 3GPP TS 33.102: "Technical specification group services and system aspects; Proximity-based services (ProSe); 3G security; Security architecture", Release 14, 2017. 